\title{
A few remarks on hyperbolic systems with Zygmund in time coefficients
}

\author{
Francesco Fanelli
}

In honour of Guy Métivier in occasion of his 65th birthday

"Towering, genius disdains a beaten path. It seeks regions hitherto unexplored."

\begin{abstract}
This note deals with first order hyperbolic systems with constant multiplicities. We assume that the coefficients of the operator depend just on the time variable, and they verify Zygmund-type regularity conditions. For such operators, well-posedness in the framework of Sobolev spaces is known to hold, possibly with a finite loss of derivatives. The main purpose of the present paper is to establish finite propagation speed, which is the key to build up a local theory.

The argument of the proof relies on showing fine estimates about the propagation of the support of the solution: this is achieved by passing in Fourier variables and applying Paley-Wiener theorem. In particular, our approach is specific of the timedependent case, and it cannot be extended to operators whose coefficients depend also on the space variables.
\end{abstract}

\section{Introduction}

The present paper sets in the context of the analysis of linear first order hyperbolic systems of the form

$$
P u(t, x)=\partial_{t} u(t, x)+\sum_{j=1}^{n} A_{j}(t, x) \partial_{j} u(t, x),
$$

for which one assumes low regularity hypotheses on the coefficients.

Francesco Fanelli

Institut Camille Jordan - CNRS UMR 5208, Université Claude Bernard - Lyon 1; 48, Boulevard du 11 novembre 1918 - 69622 Villeurbanne cedex, FRANCE; e-mail: fanelli@math.univ-lyon1.fr 
Before entering into the details of the question, for which we refer to Subsections 1.2 and 1.3 , let us explain our main motivations.

\subsection{Motivations}

The importance of such a study comes first of all from modelling purposes. Indeed, several physical systems can be described, from the mathematical viewpoint, by hyperbolic problems with variable, and especially non-smooth, coefficients.

This is the case, for instance, of propagation of waves (signals) in strongly heterogeneous media, or in media presenting a non-Euclidean metric. Many are the situations, from e.g. physics to engineering, where this kind of phenomena appears. At the mathematical level, the model is often described by a scalar wave equation of the form

$$
W u(t, x):=\partial_{t}^{2} u(t, x)-\sum_{j, k=1}^{n} \partial_{j}\left(a_{j k}(t, x) \partial_{k} u(t, x)\right)
$$

which was extensively studied so far (see e.g. the pioneering paper [8], or more recent works [13], [14], [31]). We will present in the next paragraph a short overview of results and additional references.

For the time being, let us stress that all these works highlighted the influence of the lack of regularity of the coefficients on the time evolution of the system. In particular, strange (sometimes pathological) phenomena may be produced: namely, no matter how smooth the initial data are, a loss of regularity of the solution may occur, and this loss is usually increasing when time goes by. Such an occurrence affects both the well-posedness theory and the observability and control of waves, and it makes some properties unclear to hold for operator (2), if the regularity of its coefficients is too weak.

On the other hand, linear hyperbolic operators with variable non-regular coefficients may arise also in the study of singular perturbation problems.

Some examples come from models of fluid mechanics: we refer e.g. to [29] for the incompressible limit of the compressible Euler equations, to [24] for the fast rotation limit of a Navier-Stokes system with Coriolis force, and to [22] for a combination of the two previous effects for compressible Navier-Stokes equations with Earth rotation.

As a matter of fact, in this kind of problems, when general ill-prepared initial data are considered, fast oscillations in time are produced by the component of the solutions which belongs to the orthogonal complement of the kernel of the penalized operator. Usually, the propagation of these oscillations is governed by a linear system of waves: for instance, for the incompressible limit one speaks about acoustic waves, about Rossby waves for the high rotation limit.

Depending on the working hypotheses, the singular perturbation operator may present variable coefficients. If we come back to the previous examples, this is what 
happens for the incompressible asymptotics, whenever the limit density profile is taken to be non-constant (e.g. if non-monotone pressure laws are considered, or if additional forces act on the system). Or even for the case of fluids under the effects of fast Earth rotation: indeed, it is well-known that the strenght of the Coriolis force depends on the latitude, and then it is physically relevant to consider a variable coefficient in front of the rotation operator.

In the former instance described above, the regularity of the variable coefficients is dictated by the problem, and in particular it may be very low depending on the considered functional framework (e.g. for weak solutions, or solutions in critical spaces). In the latter case, we remark that physical models usually take smooth variations of the rotation axis; nevertheless, heterogeneity and high irregularity are very common issues in nature: think for instance to jumps and discontinuities, fractures, strong concentrations and fast oscillations. It is then relevant to include also these effects in our description of the processes, not only for a purely mathematical taste.

In the light of what we have just said, it appears clear the importance of being able to deal with coefficients of limited smoothness in singular perturbation operators.

Finally, the study of linear operators may find applications also at the non-linear level. This is the case e.g. of the analysis of transport equations with low regularity coefficients, which may be seen as a sort of toy-model for purely hyperbolic problems. Nowadays there is a really broad literature devoted to this subject, which is not completely well-understood yet: here we limit ourselves to quote the fundamental papers [19]-[1], and we refer to Chapter 3 of [4] for a review of previous results and additional important references.

The analysis developed on the linear model is a fundamental step, for instance, in the understanding of the 2-D incompressible Euler equations (written here in the vorticity formulation)

$$
\left\{\begin{array}{l}
\partial_{t} \omega+u \cdot \nabla \omega=0 \\
\omega=\nabla \times u \\
\operatorname{div} u=0
\end{array}\right.
$$

in a low regularity functional framework, like e.g. in Yudovich theory (see [34]) or in Delort theory (see [18]).

For example, in the former setting, i.e. when the initial vorticity $\omega_{0} \in L^{p}\left(\mathbb{R}^{2}\right) \cap$ $L^{\infty}\left(\mathbb{R}^{2}\right)$ for some $p \geq 1$, then (see e.g. paper [3]) the corresponding velocity field $u$ is just log-Lipschitz in space, and this fact causes a loss of regularity for the solution $\omega$ in Hölder or Sobolev norms, similarly to what happens for wave equations (2). We refer to [6] and [4] (see Chapter 7) for a more detailed review on this subject.

In this context, let us mention that, despite the huge number of works devoted to this topic, there are still unclear questions. Just to mention one among them, we point out that, to the best of our knowledge, the analogue of Yudovich theory is completely unknown to hold for non-homogeneous inviscid fluids, i.e. for fluids which present variable density. By the structure of the system (see e.g. papers [16][17]), it seems to be important to have a good understanding and a "strong" theory 
for linear operators, in order to be able to deal with the lack of smoothness of the coefficients (and consequently of the solutions) in a sharp way.

\subsection{Background: a quick overview}

Now, we want to give a short summary on previous results about hyperbolic operators with coefficients having limited smoothness. As said above, this lack of regularity may affect the evolution of the system, producing pathological effects already at the linear level.

For the case of the scalar wave operators $W$, defined in (2), we refer e.g. to [8], [13], [7], [14] and [31] for some results; recent developments, as well as additional references, can be found in [9]-[10]. For the case of first order systems (1), here we quote works [27] (see Chapters 2-3-7), [28], [15], [11] and [12].

Without entering into the details of the specific results, let us make some general comments. The first issue to point out is that the Lipschitz regularity represents a quite rigid threshold for the well-posedness of hyperbolic problems to hold in the classical energy space $L^{2}$ with no loss of derivatives. This means that the solution propagates the regularity of the initial data and external force at any time $t$.

Below the Lipschitz condition, things go worse and we have to remark that a loss of smoothness produces for the solution during the time evolution, no matter how smooth the initial data and the external force are. This phenomenon has drastic consequences in the $\left(L^{2}\right.$ or $\left.\mathscr{C}^{\infty}\right)$ well-posedness of the Cauchy problem, which can be recovered only admitting a loss of a finite number of derivatives. But this is not all: explicit counterexamples establish the sharpness (if one measures regularity just by the modulus of continuity) of the Lipschitz and log-Lipschitz assumptions, for the $L^{2}$ and the $\mathscr{C}^{\infty}$ well-posedness respectively. In particular, these counterexamples show that, for too irregular coefficients, the loss of derivatives can be infinite, precluding any possibility of recovering well-posedness, even in a weak sense.

To conclude, let us mention that similar phenomena have been proved to occur, for wave operators (2), also at the level of observability and controllability. We refer e.g. to works [2], [5], [23], [21] and [33].

In this picture, of course there are specific differences between the case of scalar equations (2) and of systems (1).

Here we limit ourselves to mention that, for operator $P$, a fundamental hypothesis is the microlocal symmetrizability of the system, in the sense of Métivier (see Definition 2 below). Roughly speaking, this means that there exists a scalar product (say) $S$, depending both on $(t, x)$ and on the dual variable $\xi \neq 0$, with respect to which the operator becomes self-adjoint, and then "classical" energy estimates work. For example, strictly hyperbolic systems, or more in general hyperbolic systems with constant multiplicities, are microlocally symmetrizable (at least for regular coefficients, see also the discussion below), because they are smoothly diagonalizable.

The dependence of $S$ on $\xi$ is usually assumed to be very smooth (i.e. $\mathscr{C}^{\infty}$ ), while various regularity hypotheses can be considered with respect to the time and space 
variables: accordingly, one speaks about bounded (or continuous, or Lipschitz, and so on) symmetrizer.

By a result of Ivrii and Petkov (see [25]), the existence of a bounded microlocal symmetrizer is a necessary condition for the Cauchy problem for $P$ to be well-posed in $\mathscr{C}^{\infty}$. As for sufficient conditions, the question is still widely open; the main feature to point out is that regularity hypotheses are needed both on the coefficients $A_{j}$ of the operator and the symmetrizer $S$. This is not just a technical requirement: the counterexamples we mentioned above (see e.g. [15]) prove that this is a key point which cannot be avoided.

Paper [31] by Tarama changed a little bit the point of view about this matter. There, the author dealt with the case of the scalar operator $W$, defined in (2): for coefficients only depending on time, namely $a_{j k}=a_{j k}(t)$ for all $(j, k)$, he introduced Zygmund-type regularity hypotheses.

These are somehow second order conditions, made on the symmetric difference of the function rather than on its modulus of continuity, and they are weaker than the corresponding ones formulated on the first difference. The special issue is that they are still suitable for well-posedness of hyperbolic Cauchy problems, with the same kind of loss which would pertain to the latter conditions. So, for pure Zygmund hypothesis Tarama proved well-posedness in any $H^{s}$ with no loss of derivatives, while for log-Zygmund hypothesis he showed energy estimates with a finite loss of derivatives.

In order to get the improvement from Lipschitz to Zygmund conditions, the main idea of Tarama was to compensate the worse behaviour of the coefficients by introducing a lower order corrector in the definition of the energy, in order to produce special algebraic cancellations in the energy estimates.

In passing, we mention that, in [9] and [10], Zygmund assumptions were exploited further for operators with coefficients $a_{j k}(t, x)$ also depending on the space variable, in combination with suitable regularity hypotheses with respect to $x$. We refer to those papers for the precise results and for additional comments.

Inspired by work [31], in [11] we focused on the special case of first order systems with just time dependent coefficients,

$$
L u(t, x)=\partial_{t} u(t, x)+\sum_{j=1}^{n} A_{j}(t) \partial_{j} u(t, x),
$$

for which we formulated Zygmund and log-Zygmund type assumptions. For reasons which will appear clear in a while, however, we dismissed the hypothesis of microlocal symmetrizability of the system, and we supposed $L$ to be hyperbolic with constant multiplicities.

Analogously to what happens for operator $W$, for pure Zygmund conditions we proved well-posedness in any $H^{s}$ with no loss of derivatives, while we proved energy estimates with finite loss of derivatives, increasing in time, under a log-Zygmund regularity hypothesis.

The idea of the proof adopted in [11] was the same as in [31]: Tarama's cancellations in energy estimates are a fundamental ingredient in this context, and they 
cannot be bypassed. Hence, knowing a priori the existence of a microlocal symmetrizer is out of use: the main challange, here, is to build up a suitable microlocal symmetrizer for making estimates work. This is precisely what was done in [11]: in the end, we constructed a family of approximate microlocal symmetrizers (but in a somehow weaker sense), which included lower order correctors in their definition, in order to reproduce Tarama's cancellations. In particular, one has to remark that the construction is based on the choice of an explicit basis of eigenvectors: then, it is just local in $(t, \xi)$, and one has to forget about global regularity in $\xi$. In addition, we stress the fact that we do not have a true symmetrizer for our system, but just a family of approximate symmetrizers.

These facts make some points unclear, or at least less straightforward, in the analysis of first order hyperbolic systems, when assumptions of Zygmund type are formulated.

\subsection{Contents of the paper}

The present note is placed in such a theoretical context. We are going to consider operators $L$ as defined in (3), which are hyperbolic with constant multiplicities and whose coefficients verify Zygmund type regularity conditions. Our main intent is to complete the analysis developed in [11], mostly concerning a couple of aspects.

As just mentioned above, in that paper the construction of a family of suitable approximate symmetrizers was based on an explicit choice of a basis of eigenvectors. In particular, this construction is local, and it is not possible to get a unique global definition (for instance, by partition of unity) preserving the regularity in time and Fourier variables. As a matter of fact, it is better to work locally, and then carefully glue all the estimates together in the end.

This second step of the proof, i.e. how to combine all the local inequalities together, was not really detailed in paper [11]. Our first goal here is to provide a rigorous scheme in order to find global energy estimates, clarifying better this last point of the analysis in the above mentioned work.

However, the principal purpose of the present manuscript is to address local questions for the operators under consideration, which were not considered in [11]. In particular, we are interested in showing finite propagation speed, which is the key property in order to build up a local theory.

For establishing finite speed of propagation, a classical approach is based on convexification arguments, combined with a global $L^{2}$ well-posedness result. This strategy was adopted in [28] for operator (1) with Lipschitz coefficients and symmetrizer. A crucial point, there, was to show that the assumed hypotheses are invariant by change of variables, and in particular of the timelike direction. Then, finite speed of propagation and local uniqueness follow; in addition, the previous property allows for a sharp description of the propagation of the supports, like in [26] and [30]. Remark that the results in these last papers still require some smoothness assumptions on the coefficients, besides asking to know a priori a local uniqueness 
property to hold at any spacelike hypersurface. We also point out that, in [28], regularity of the symmetrizer on $\xi$ can be considerably weakened to Lipschitz, if the $A_{j}$ 's enjoy stronger $W^{2, \infty}$ regularity in $(t, x)$.

The same scheme (finite propagation speed by convexification) is followed also in [12] for operator $P$, defined in (1), with log-Lipschitz coefficients, under the hypothesis that it admits a symmetrizer which is log-Lipschitz in $(t, x)$ and smooth in $\xi \neq 0$. Regularity in $\xi$ is a key ingredient, since the well-posedness issue (with a finite loss of derivatives) exploits paradifferential calculus in a crucial way. Moreover, there many efforts are devoted to giving sense to the local Cauchy problem, due to the loss of derivatives in the energy estimates (a difficulty which was already encountered in [14] for wave equations).

In paper [20], instead, we restricted our attention to operator (3) with coefficients depending only on time, assuming moreover the existence of a microlocal symmetrizer. By use of elementary methods (see below for the details), we established finite propagation speed under very weak regularity assumptions on the matrices $A_{j}$ and on $S$ : roughly speaking, either $A_{j} \in L^{1}([0, T])$ and global continuity of $S$ on $(t, \xi)$, or $A_{j}$ 's and $S$ uniformly bounded. On the other hand, referring to the above mentioned works [28]-[12], one has to remark that the analysis of [20] applies to operators with just time dependent coefficients, and hence, in particular, one also loses the invariance by change of coordinates.

Here, we will adopt the same approach as in [20], which in turn was strongly inspired by the analysis of [8] for scalar wave equations (2). More precisely, our strategy is based on fine estimates on the growth of the solution in Fourier variables and on the application of Paley-Wiener Theorem; at this stage, we exploit the energy estimates we show in the first part of the paper in a fundamental way. In analogy with what happens for operators $W$ (see [8]) and what is proved in [20], we see that it is still the $L^{1}([0, T])$-norm of the coefficients which enters into play in the propagation of the support of the initial data.

As a conclusion, let us point out that the analysis of [20] does not apply straight away to our setting here. Indeed, it is true that Zygmund regularity would be more than enough, if compared with the regularity one asks for in the results of [20]. Nevertheless, here we do not dispose of a unique global microlocal symmetrizer, but only of a family of local symmetrizers, which cannot be glued together and for which smoothness hypotheses fail. Moreover, these scalar products are just approximate symmetrizers, in the sense that they symmetrize a perturbed operator (say) $L_{\mathcal{E}}$, and not the original $L$ in (3).

\section{Structure of the paper}

Before going on, let us present an overview of the paper.

In the next section we will set our working hypotheses, and we will state our main result, about finite propagation speed for operators (3) with Zygmund type coefficients. In Section 3 we recall the well-posedness result in Sobolev spaces for this kind of operators, showing a sketch of the proof of the energy estimates which were 
presented in [11]. Besides, we will detail the argument in order to pass from local to global in time estimates. Finally, in Section 4 we will prove the finite propagation speed property.

\section{Notations}

We introduce here some notations which will be freely used in the text.

Let the field $\mathbb{F}$ be $\mathbb{R}$ or $\mathbb{C}$, and fix a number $n \geq 1$. Let $u$ be a function defined on $\mathbb{F}^{n}$ : the variable, if real, will be usually called $x$, while $z$ if complex. Correspondingly, we denote by $\xi \in \mathbb{R}^{n}$ or $\zeta \in \mathbb{C}^{n}$ the respective dual variables. We will also use the decompositions $z=x+i y$ and $\zeta=\xi+i \eta$, with both $(x, \xi)$ and $(y, \eta)$ in $\mathbb{R}^{2 n}$.

Given two vectors $v$ and $w$ in $\mathbb{C}^{m}$, we will denote by $v \cdot w$ the usual hermitian product in $\mathbb{C}^{m}$ and by $|v|$ the usual norm of a vector in $\mathbb{C}^{m}$ : namely,

$$
v \cdot w=\sum_{j=1}^{m} v_{j} \overline{w_{j}} \quad \text { and } \quad|v|^{2}=v \cdot v .
$$

On the contrary, given an infinite-dimensional Banach space $X$, we denote by $\|\cdot\|_{X}$ its norm and, if $X$ is Hilbert, by $(\cdot, \cdot)_{X}$ its scalar product.

The symbol $\mathscr{M}_{m}(\mathbb{F})$ refers to the set of all $m \times m$ matrices whose components belong to $\mathbb{F}$, equipped with the norm $|\cdot| \mathscr{M}$ defined by

$$
|A|_{\mathscr{M}}:=\sup _{|v|=1}|A v| \equiv \sup _{v \neq 0} \frac{|A v|}{|v|} .
$$

If $A$ is self-adjoint (more in general, if it is a normal matrix), we also have

$$
|A|_{\mathscr{M}} \equiv \sup _{|v|=1}|A v \cdot v|
$$

Finally, given two self-adjoint matrices $A$ and $B$ belonging to $\mathscr{M}_{m}(\mathbb{C})$, we say that $A \leq B$ if the inequality $A v \cdot v \leq B v \cdot v$ holds true for all $v \in \mathbb{C}^{m}$.

\section{The main result}

We present here the main result of this paper, concerning finite propagation speed property for hyperbolic operators with Zygmund-type coefficients.

First of all, let us state our working assumptions. 


\subsection{Basic definitions and hypotheses}

On an infinite strip $[0, T] \times \mathbb{R}^{n}$, for some time $T>0$ and $n \geq 1$, we consider the $m \times m$ (with $m \geq 1$ ) linear first order system

$$
L u(t, x)=\partial_{t} u(t, x)+\sum_{j=1}^{n} A_{j}(t) \partial_{j} u(t, x) .
$$

Namely, we suppose $u(t, x) \in \mathbb{R}^{m}$ and, for all $1 \leq j \leq n$, the matrices $A_{j}(t) \in \mathscr{M}_{m}(\mathbb{R})$.

We immediately introduce the symbol $A$ associated to the operator $L$ : for all $(t, \xi) \in[0, T] \times \mathbb{R}^{n}$

$$
A(t, \xi):=\sum_{j=1}^{n} \xi^{j} A_{j}(t) .
$$

Then, for all $(t, \xi)$, the matrix $A(t, \xi)$ belongs to $\mathscr{M}_{m}(\mathbb{R})$. Let $\left(\lambda_{j}(t, \xi)\right)_{1 \leq j \leq m} \subset \mathbb{C}$ denote the family of its eigenvalues at any point $(t, \xi)$.

We recall the following definitions (see e.g. [27], Chapter 2).

Definition 1. (i) We say that system (4) is hyperbolic if, for all $t \in[0, T]$ and all $\xi \neq 0$, the eigenvalues of $A(t, \xi)$ are all real: $\left(\lambda_{j}\right)_{1 \leq j \leq m} \subset \mathbb{R}$.

(ii) System (4) is hyperbolic with constant multiplicities if, for all $t \in[0, T]$ and all $\xi \neq 0$, the eigenvalues of $A(t, \xi)$ are real and semi-simple, with constant multiplicities.

We point out that a (possibly complex) eigenvalue is called semi-simple if its algebraic and geometric multiplicities coincide; a matrix is semi-simple if it is diagonalizable in the complex sense.

A particular case of hyperbolicity with constant multiplicities is when the operator is strictly hyperbolic, i.e. when all the eigenvalues are real and distinct (constant multiplicites equal to 1 ).

In what follows, we are going to consider first order hyperbolic systems with constant multiplicities. However, let us recall also the notion of microlocal symmetrizability in the sense of Métivier (see [27], Chapter 7). The word "uniformly" here below refers to $(t, x) \in[0, T] \times \mathbb{R}^{n}$ (see also Section 4 of [28]).

Definition 2. Operator $L$, defined in (4), is a uniformly microlocally symmetrizable hyperbolic system if there exists a $m \times m$ matrix $S(t, \xi)$, homogeneous of degree 0 in $\xi$, such that:

- for almost every $t$, the map $\xi \mapsto S(t, \xi)$ is $\mathscr{C}^{\infty}$ for $\xi \neq 0$;

- for any point $(t, \xi)$, the matrix $S(t, \xi)$ is self-adjoint;

- there exist constants $0<\lambda \leq \Lambda$ such that $\lambda \operatorname{Id} \leq S(t, \xi) \leq \Lambda$ Id for any $(t, \xi)$;

- for any point $(t, \xi)$, the matrix $S(t, \xi) A(t, \xi)$ is self-adjoint.

The matrix valued function $S(t, \xi)$ is called a (bounded) microlocal symmetrizer for system (4). 
Remark 1. Microlocal symmetrizability is a fundamental notion in the theory of first order hyperbolic systems. Nevertheless, as explained in the Introduction, it is out of use under the assumptions we are going to impose on the regularity of our coefficients. This is why in the present paper we will consider hyperbolic systems with constant multiplicities.

Now, we turn our attention to the coefficients of $L$ : we assume just Zygmundtype conditions, i.e. suitable growth bounds on the symmetric difference at every point $t \in[0, T]$. As done in [11], we measure these buonds in $L^{p}$ norms, for any $1 \leq p \leq+\infty$, and we can allow also a logarithmic loss.

More precisely, fixed $\ell \in\{0,1\}$, we suppose that there exist a $p \in[1,+\infty]$ and a constant $K>0$ such that, for all $1 \leq j \leq n$ and all $0<\tau<T / 2$,

$$
\left\|A_{j}(\cdot+\tau)+A_{j}(\cdot-\tau)-2 A_{j}(\cdot)\right\|_{L^{p}([\tau, T-\tau] ; \mathscr{M})} \leq K \tau \log ^{\ell}\left(1+\frac{1}{\tau}\right) .
$$

The case $\ell=0$ corresponds to the Zygmund regularity hypothesis, the case $\ell=1$ to the log-Zygmund regularity hypothesis.

In particular, the previous condition implies that each component of the matrices $A_{j}$ verifies the same integral Zygmund condition (as real-valued functions over $[0, T])$.

Remark 2. When $p>1$, estimate (6) is already enough to ensure a uniform pointwise bound:

$$
\sum_{j=1}^{n} \sup _{t \in[0, T]}\left|A_{j}(t)\right|_{\mathscr{M}} \leq C_{0}
$$

We refer to Section 3 of [11] for further properties of Zygmund and log-Zygmund classes, as well as for their characterization as (possibly logarithmic) Besov spaces.

\subsection{Finite speed of propagation}

In this paper, our main goal is to investigate local questions for microlocally symmetrizable first order hyperbolic systems with Zygmund-type coefficients.

The most important step is to establish local existence and finite propagation speed for our operators. Then, the other results (e.g. domain of dependence, local uniqueness) will follow in a quite standard way.

First of all, let us recall a well-posedness result in the class of Sobolev spaces.

Theorem 1. Let us consider the first-order system (4), and let us assume it to be hyperbolic with constant multiplicities. Suppose moreover that the coefficients $\left(A_{j}\right)_{1 \leq j \leq n}$ satisfy condition (6), for some $1<p \leq+\infty$. Set $\theta:=1-1 / p$. Fixed $s \in \mathbb{R}$, assume that $u_{0} \in H^{s}$ and $f \in L^{1}\left([0, T] ; H^{s}\right)$.

Then, there exists a unique solution u to the Cauchy problem 


$$
\left\{\begin{array}{l}
L u=f \\
u_{\mid t=0}=u_{0}
\end{array}\right.
$$

which belongs to the space $\mathscr{C}\left([0, T] ; H^{s}\right)$ if $\ell=0$, and to the space $\mathscr{C}\left([0, T] ; H^{s-\beta(\cdot)}\right)$ in the case $\ell=1$, where the function $\beta$ is defined by $\beta(t)=\beta_{0} t^{\theta}$, for a suitable constant $\beta_{0}>0$ just depending on $K$ in (6).

In the previous statement, the property $u \in \mathscr{C}\left([0, T] ; H^{s-\beta(\cdot)}\right)$ means that, for any $t \in[0, T]$, one has

$$
u(t) \in H^{s-\beta(t)},
$$

and the map $t \mapsto u(t)$ is continuous between the respective functional spaces.

Theorem 1 was proved in [11]. However, we will give a sketch of its proof in Section 3, where we postpone also some important remarks. Moreover, we will also clarify some technical points which were not detailed in the above mentioned paper.

Now, let us focus on the finite speed of propagation property for our operator. Here below, as in the rest of the paper, we adopt the following notation: fixed some $r>0$, we set $B(r)$ to be the ball of $\mathbb{R}^{n}$ centered in the origin and of radius $r$.

The main result of this note is the next statement.

Theorem 2. Let us consider the first-order system (4), and let us assume it to be hyperbolic with constant multiplicities. Suppose moreover that the coefficients $\left(A_{j}\right)_{1 \leq j \leq n}$ satisfy condition (6), for some $1<p \leq+\infty$ and for $\ell=1$.

Fixed $s \in \mathbb{R}$, assume that $u_{0} \in H^{s}$ and $f \in L^{1}\left([0, T] ; H^{s}\right)$, and let $u$ be the solution to (8) given by Theorem 1.

Finally, assume that $\operatorname{supp} u_{0}$ and $\operatorname{supp} f(t)$ (for almost every $t$ ) are contained in the ball $B\left(r_{0}\right)$, for some radius $r_{0}>0$.

Then, there exists a constant $\mathbb{K}>0$, depending just on the constant $K$ in (6), such that, if we define the quantities

$$
\alpha(t):=\sum_{j=1}^{n}\left|A_{j}(t)\right|_{\mathscr{M}} \quad \text { and } \quad r(t):=r_{0}+\mathbb{K} \sqrt{\Lambda} \int_{0}^{t} \alpha(\tau) d \tau,
$$

for all $t \in[0, T]$ one has that $\operatorname{supp} u(t) \subset B(r(t))$.

We have decided to present the result just for $\ell=1$ for simplicity: it goes without saying that an analogous result holds true a fortiori in the case $\ell=0$.

\section{Well-posedness in Sobolev classes}

Before showing the proof of Theorem 2, we turn our attention to the well-posedness theory for our operators in Sobolev spaces. This was essentially established in the above mentioned paper [11]: however, for the sake of completeness we sketch here most of the details. 
To begin with, we recall some general facts, which are needed in our analysis. This part is essentially taken from Section 1 of [8]. We present also the main energy estimate, as stated in [11]: see Theorem 4, which will be commented in detail in Subsection 3.2. In Subsection 3.3 we show a sketch of its proof, which will be completed in Paragraph 3.4 passing from local to global estimates.

\subsection{The basic energy estimate}

First of all, let us introduce additional notations for some relevant functional spaces. Let $\Omega$ be an open subset of $\mathbb{R}^{n}$; then:

- $\mathscr{H}$ denotes the space of entire functions on $\mathbb{R}^{n}$;

- $\mathscr{A}(\Omega)$ denotes the space of real analytic functions on $\Omega$;

- $\mathscr{H}^{\prime}$ is the space of holomorphic functionals over $\mathbb{C}^{n}$;

- $\mathscr{A}^{\prime}(\Omega)$ is the space of real analytic functionals over $\Omega$.

When $\Omega=\mathbb{R}^{n}$, we will omit it from the notation. In addition, we will indicate by $\operatorname{supp} u$ the support of a functional $u \in \mathscr{A}^{\prime}(\Omega)$.

We recall that, given a compact set $K \subset \Omega$, we say that supp $u \subset K$ if, for all open neighborhood $U \subset \Omega$ of $K$ and all sequence $\left(f^{k}\right)_{k} \subset \mathscr{A}(\Omega)$ such that $f^{k} \rightarrow 0$ in $\mathscr{A}(U)$, then one has $<u, f^{k}>\longrightarrow 0$.

The symbol $<\cdot, \cdot>\mathscr{H}^{\prime} \times \mathscr{H}$ refers to the duality product for the space $\mathscr{H}^{\prime} \times \mathscr{H}$, and an analogous notation will be used for $\mathscr{A}^{\prime}(\Omega) \times \mathscr{A}(\Omega)$. The spaces in the subscript will be omitted whenever they are evident from the context.

For a functional $u \in \mathscr{H}^{\prime}$, we denote by $\widehat{u}$ its Fourier transform: for all $(z, \zeta) \in \mathbb{C}^{2 n}$, one has

$$
\widehat{u}(\zeta):=<u, h_{\zeta}>, \quad \text { with } \quad h_{\zeta}(z):=\exp (-i \zeta \cdot z) .
$$

For $u \in L^{1}\left([0, T] ; \mathscr{H}^{\prime}\right)$, we write $\widehat{u}(t, \zeta)=<u(t), h_{\zeta}>$ for almost every $t$.

We recall that a real analytic functional $u$ is real-valued if, for any real analytic function $\varphi$ over $\mathbb{R}^{n}$, the quantity $\langle u, \varphi\rangle \in \mathbb{R}$. Whenever $u$ is compactly supported, this is equivalent to require that $\widehat{u}(-\xi)=\overline{\widehat{u}(\xi)}$ for all $\xi \in \mathbb{R}^{n}$.

This having been clarified, let us present a very general existence and uniqueness result, in the same spirit of the celebrated Cauchy-Kovalevska Theorem. We refer to Theorem 1 of [8] and Theorem 17.2 of [32] for its proof.

Theorem 3. Let $L$ be the operator defined in (4), and suppose that its coefficients $\left(A_{j}\right)_{j}$ belong to the space $L^{1}\left([0, T] ; \mathscr{M}_{m}(\mathbb{R})\right)$.

Then, for any $u_{0} \in \mathscr{H}^{\prime}$ and $f \in L^{1}\left([0, T] ; \mathscr{H}^{\prime}\right)$, the Cauchy problem (8) admits a unique solution $u \in \mathscr{C}\left([0, T] ; \mathscr{H}^{\prime}\right)$.

The proof is analogous to the one given in Appendix B of [8], and it is based on a Picard iteration scheme. Actually, in that paper the result is stated for scalar wave operators, but the proof consists in recasting the equation as a first order system. 
Here, the only difference is the regularity in time of the solution, which is obtained by an inspection of the equation $L u=f$.

Remark 3. We remark that no hyperbolicity hypotheses are needed on the system, for the general existence result in the space of holomorphic functionals.

Thanks to the previous statement and Paley-Wiener theorem, the proof of Theorem 1 reduces to find suitable bounds on the growth of the $H^{s}$ norm of the solution to problem (8). This was done in [11]: more precisely, one has the following result.

Theorem 4. Let us consider the first order system (4), and let us assume it to be hyperbolic with constant multiplicities.

Suppose moreover that the coefficients $\left(A_{j}\right)_{1 \leq j \leq n}$ satisfy condition (6), for some $1<p \leq+\infty$. Finally, set $\theta:=1-1 / p$.

(a) Let $\ell=0$.

Then, for all $s \in \mathbb{R}$, there exist positive constants $C_{1}, C_{2}$ (just depending on $s$ and on $K$ ) such that the estimate

$$
\sup _{t \in[0, T]}\|u(t)\|_{H^{s}} \leq C_{1} e^{C_{2}\left(T+T^{\theta}\right)}\left(\|u(0)\|_{H^{s}}+\int_{0}^{T}\|L u(\tau)\|_{H^{s}} d \tau\right)
$$

holds true for any $u \in \mathscr{C}^{1}\left([0, T] ; H^{\infty}\left(\mathbb{R}^{n} ; \mathbb{R}^{m}\right)\right)$.

(b) Let $\ell=1$.

Then, for all $s \in \mathbb{R}$, there exist a positive constants $C_{1}, C_{2}$ (depending on $s$ and on $K$ ) and $a \beta_{0}>0$ (depending just on $K$ ) such that, defined $\beta(t):=\beta_{0} t^{\theta}$, then, for any $u \in \mathscr{C}^{1}\left([0, T] ; H^{\infty}\left(\mathbb{R}^{n} ; \mathbb{R}^{m}\right)\right)$, the estimate

$$
\|u(t)\|_{H^{s-\beta(t)}} \leq C_{1} e^{C_{2} t}\left(\|u(0)\|_{H^{s}}+\int_{0}^{t}\|L u(\tau)\|_{H^{s-\beta(t)+\beta(t-\tau)}} d \tau\right)
$$

holds true for all time $t \in[0, T]$.

Before showing the proof of Theorem 4, some remarks are in order.

\subsection{Some remarks}

We list here several important remarks concerning the previous statement.

1) Well-posedness in Sobolev classes

The estimates of Theorem 4 entail well-posedness in any $H^{s}$ for pure Zygmund coefficients, and well-posedness in $H^{\infty}$ with finite loss of derivatives for logZygmund coefficients.

2) Hypothesis on $p$

In the previous statement, in general $p$ has to be strictly greater than 1 . This requirement is needed several times in the course of the proof. 
However, in the particular case of systems coming from a second order scalar equation, the weakest condition $p=1$ is still suitable to recover energy estimates, when combined with an additional $L^{\infty}$ assumption on the coefficients.

3) On the hyperbolicity hypothesis

In Theorem 4 above, we have not assumed our operator to be microlocally symmetrizable, but just hyperbolic with constant multiplicities (which is well-known to be a stronger hypothesis). Recall also Remark 1 above.

This is due to the very low regularity assumptions on the coefficients of the operator. More precisely, under these conditions, the existence of a symmetrizer, which inherits the same smoothness in time of the matrices $A_{j}$, is not enough to find suitable energy estimates, due to an extra loss of derivatives in the energy estimates.

Then, roughly speaking, one needs to introduce a lower order corrector in the definition of the energy, in order to compensate such an extra loss. This strategy follows the original idea of Tarama in [31] for scalar wave operators. So, the difficulty of this case relies in constructing a suitable microlocal symmetrizer (say) $S$ for $L$.

4) Finding a symmetrizer for $L$

We have to point out that the scalar product $S$, which we are going to build in the next subsection, is not a microlocal symmetrizer in the very precise sense of Definition 2 (see Remark 5 below).

Furthermore, our construction is only local in $(t, \xi)$, since it is based on an explicit choice of a basis of eigenvectors for the matrix symbol $A(t, \xi)$. It is not straightforward to generalize our arguments in order to find estimates on the whole $[0, T] \times \mathbb{R}^{n}$.

We will be more clear about these points in the next subsection, when we recall the main ideas of the proof. See in particular Paragraph 3.3.2, where we present the construction and we point out further important aspects of the question.

\subsection{Sketch of the proof}

We revise here the key issues of the proof to Theorem 4 (see paper [11] for the complete arguments).

We follow a scheme which is now standard in tackling this kind of problems, and which goes back to the pioneering paper [8]. It consists of the following main steps:

1. approximation of the coefficients;

2. choice of the approximation parameter depending on the size of the frequencies;

3. definition of a suitable approximate energy, and energy estimates.

So, let us implement this program. 


\subsubsection{Approximation scheme}

The preliminary step is to define smooth approximate coefficients. Since the perturbation of a hyperbolic matrix with constant multiplicities is not hyperbolic with constant multiplicities in general, we have to proceed carefully.

Hence, first of all we diagonalize the matrix symbol $A(t, \xi)$ by writing

$$
A(t, \xi)=P(t, \xi) \Lambda(t, \xi) P^{-1}(t, \xi)
$$

where $\Lambda$ is the eigenvalue matrix and $P$ is a change of basis operator. Let us note that the column vectors of $P$ are eigenvectors for $A$.

Remark 4. By standard perturbation theory for linear operators, $\Lambda$ and $P$ keep the same regularity in time (and in $\xi$ ) of the matrix $A$. On the other hand, the matrix $P$ is defined only locally on $(t, \xi)$. We refer to Remarks 5,6 and 7 for further important comments about this point; we refer to Paragraph 3.4 below for the details of the extension of our arguments to the whole interval $[0, T]$.

For the time being, let us just point out that these local pieces are not suitable for a global definition of a unique symmetrizer on $[0, T] \times \mathbb{S}^{n-1}$ (for instance, by gluing them using partions of unity in $t$ and $\xi$ ).

So, for any fixed $\xi \in \mathbb{S}^{n-1}$ (this is enough for our scopes, because the symmetrizer will be homogeneous of degree 0 ), we have to work locally in time.

In each interval where relation (11) holds, we smooth out the eigenvectors and eigenvalues in time by convolution with a mollifier kernel. Then, we apply formula (11) again on the approximate matrices (say) $\Lambda_{\varepsilon}$ and $P_{\varepsilon}$ (where $\left.\left.\varepsilon \in\right] 0,1\right]$ is the approximation parameter) in order to define the approximate symbol $A_{\varepsilon}(t, \xi)$.

\subsubsection{Construction of the approximate symmetrizer}

Now, for any $\varepsilon$, we construct a symmetrizer for the regularized symbol $A_{\varepsilon}(t, \xi)$.

Here, we denote by $\mathscr{Z}_{p}$ the space of " $L^{p}$-Zygmund" functions (i.e. satisfying (6) with $\ell=0$, for a certain $p \in] 1,+\infty]$ ), and by $\mathscr{L} \mathscr{Z}_{p}$ the space of " $L^{p}-\log$-Zygmund" functions (verifying (6) with $\ell=1$ ). We denote by $\partial_{t} \mathscr{Z}_{p}$ the set which is obtained by differentiation in time of functions in the previous $\mathscr{Z}_{p}$ class; analogous definition for $\partial_{t} \mathscr{L} \mathscr{Z}_{p}$.

Lemma 1. Assume the hypothesis of Theorem 4, with $\ell=0$ or $\ell=1$. Fix $\xi \neq 0$ and define the approximate matrix symbol $A_{\varepsilon}$ as above.

There exist two families of $m \times m$ real-valued self-adjoint matrices, which are smooth with respect to $t$ and homogeneous of degree 0 in $\xi$, and such that:

- $\left(S_{\varepsilon}^{0}\right)_{\varepsilon}$ is bounded in $\mathscr{Z}_{p}$ (or $\mathscr{L} \mathscr{Z}_{p}$ respectively);

- the $S_{\varepsilon}^{0}$ 's are uniformly positive definite: $S_{\varepsilon}^{0} v \cdot v \geq C|v|^{2}$ for any $v \in \mathbb{C}^{m}$, for a constant $C>0$ depending just on the constant $K$ in (6);

- $\left(S_{\varepsilon}^{1}\right)_{\varepsilon}$ is bounded in $\partial_{t} \mathscr{Z}_{p}$ (or $\partial_{t} \mathscr{L} \mathscr{Z}_{p}$ respectively). 
Moreover, for any $\varepsilon \in] 0,1]$, the matrices $S_{\varepsilon}^{0}$ and $S_{\varepsilon}^{1}$ satisfy the relation

$$
\partial_{t} S_{\varepsilon}^{0} \widehat{u} \cdot \widehat{u}+2 \operatorname{Re}\left(-i|\xi|^{-1} S_{\varepsilon}^{1} A_{\varepsilon} \widehat{u} \cdot \widehat{u}\right)=R_{\varepsilon} \widehat{u} \cdot \widehat{u},
$$

where the family of remainders $\left(R_{\varepsilon}\right)_{\varepsilon}$ is bounded in $L^{\infty}\left([0, T] ; \mathscr{M}_{m}(\mathbb{R})\right)$.

Then, we define the approximate symmetrizer as

$$
S_{\varepsilon}(t, \xi):=S_{\varepsilon}^{0}(t, \xi)+|\xi|^{-1} S_{\varepsilon}^{1}(t, \xi) .
$$

We point out here that the role of $S_{\varepsilon}^{1}$ is to kill the bad terms coming from the time derivatives of the elements of $S_{\varepsilon}^{0}$ in the energy estimates. On the other side, as the second term is of lower order, the time derivatives of $S_{\varepsilon}^{1}$ will be easily controlled in terms of the energy.

At this point, we link the approximation parameter with the dual variable, following the original idea of paper [8]: we set

$$
\varepsilon=|\xi|^{-1}
$$

Then, the matrix symbol $S_{1 /|\xi|}$, defined by (13), is a microlocal symmetrizer for the approximated system

$$
L_{\varepsilon} u=\partial_{t} u+\sum_{j=1}^{n} A_{j, \varepsilon}(t) \partial_{j} u .
$$

More precisely, we have the following proposition.

Proposition 1. Let us define $S_{\varepsilon}$ by equation (13), with $S_{\varepsilon}^{0}$ and $S_{\varepsilon}^{1}$ given by Lemma 1 and $\varepsilon$ given by choice (14).

Then, $S_{1 /|\xi|}$ enjoys the following properties:

(a) $S_{1 /|\xi|}$ is self-adjoint;

(b) for any $t$ and $|\xi| \geq R_{0}$ (for a $R_{0} \geq 1$ just depending on the constants $K$ in condition (6) above), it is uniformly bounded and (uniformly) positive definite: there exist constants $0<C_{1} \leq C_{2}$ such that, for any $v \in \mathbb{C}^{m}$,

$$
C_{1}|v|^{2} \leq S_{1 /|\xi|^{v}} \cdot v \leq C_{2}|v|^{2}
$$

(c) for all $(t, \xi)$, the matrix $S_{1 /|\xi|}^{0}(t, \xi) A_{1 /|\xi|}(t, \xi)$ is self-adjoint.

We conclude this step by stating a list of important remarks.

Remark 5. We remark that $S_{\varepsilon}$ is not a microlocal symmetrizer for $L$ in the sense of Definition 2 given above.

Indeed, it differs from a classical symmetrizer for the following features:

- its construction is just local, both in $t$ and $\xi$;

- then, smoothness in $\xi$ is just local, in the conical neighborhood where the symmetrizer is defined;

- $S_{\varepsilon} A_{\varepsilon}$ is not self-adjoint: this is true just for the higher order part $S_{\varepsilon}^{0}$. 
Remark 6. As mentioned in several parts of this paper, the approximate symmetrizer is defined only locally in $(t, \xi)$.

We point out that, for our purposes, it is not suitable to use partition of unity in the $\xi$-variable in order to past all these local definitions together.

As a matter of fact, this would lead us to work (at each $\xi$ fixed) on a smaller time interval; this fact would create us some troubles in order to get global in time estimates (see Paragraph 3.4).

Remark 7. It is not even suitable to glue all the local pieces togheter (again, by partition of unity) with respect to time variable.

Indeed, we are not able to guarantee a priori that no degeneracy occurs in the intersection of two time intervals, and that we can "interpolate" the two basis of eigenvectors in a suitable way (fatal cancellations could be produced), in order to make our argument work.

Remark 8. Let us explain why, nonetheless, a local definition is enough for us.

First of all, the local construction in time is enough, thanks to the local description of the Zygmund spaces in terms of symmetric differences.

On the other hand, even if we lose a global definition of the symmetrizer in $\xi$, thanks to the local continuity and the compactness of $\mathbb{S}^{n-1}$, it is possible to see that all the constants entering in our computations can be chosen to be independent of $\xi$. We will be more explicit in Paragraph 3.4 about this issue.

\subsubsection{Energy estimates}

It remains to us to define the approximate energy and to perform energy estimates. For doing this, it is convenient to pass in the phase space, where equation (4) reads

$$
\widehat{L u}(t, \xi)=\partial_{t} \widehat{u}(t, \xi)+i A(t, \xi) \widehat{u}(t, \xi),
$$

Then, we will follow the main lines of the proof given in [11]. However, here we want to show how to get estimates on the whole $[0, T]$, in spite of our local construction. Since this point was not detailed in [11], we devote the next subsection entirely to it.

\subsection{The global in time estimate}

For each fixed $\xi \in \mathbb{R}^{n} \backslash\{0\}$, let us take a finite family of $J$ intervals (we omit the explicit dependence on $\xi$ )

$$
I_{j}:=\left[t_{j}^{0}, t_{j}^{1}\right], \quad \text { for } \quad 1 \leq j \leq J,
$$

such that the following conditions are verified: 
- for all $j \in[1, J-1]$, one has $t_{j}^{0}<t_{j+1}^{0} \leq t_{j}^{1}<t_{j+1}^{1}$;

- $t_{1}^{0}=0$ and $t_{J}^{1}=T$, and $\cup_{j=1}^{J} I_{j}=[0, T]$

- each $I_{j}$ intersects just the two intervals next to it, namely $I_{j} \cap I_{k}=\emptyset$ if $|j-k| \geq 2$ (up to refine our intervals, we can always assume this fact);

- in each $\{\xi\} \times I_{j}$, a basis of eigenvectors for $A(t, \xi)$ is fixed, so that decomposition (11) and the subsequent construction hold true.

We notice that, actually, the set of intervals $\left(I_{j}\right)_{j}$ depends just on the direction of $\xi$, namely on $v=\xi /|\xi|$. Furthermore, by compactness, the construction can be made uniform for all $v^{\prime}$ varying in an open neighborhood $U \subset \mathbb{S}^{n-1}$ of $v$.

We have to remark that the number of intervals $J=J(\xi)$ depends on the open set $U \subset \mathbb{S}^{n-1}$ which contains $\xi$. Nonetheless, by compactness of the unitary sphere, we can bound all these $J(\xi)$ 's by a unique quantity $J_{M}$.

On the other hand, working as explained above on each set $U \times I_{j}$, we can construct local approximated symmetrizers

$$
S_{j, \varepsilon}(t, \xi)=S_{j, \varepsilon}^{0}(t, \xi)+|\xi|^{-1} S_{j, \varepsilon}^{1}(t, \xi), \quad \text { with } \quad \varepsilon=|\xi|^{-1},
$$

satisfying the properties stated in Lemma 1 and Proposition 1 on $U \times I_{j}$.

In particular, since $\mathbb{S}^{n-1}$ is compact, it is easy to deduce that the constants $C_{1}$ and $C_{2}$ appearing in Proposition 1 can be taken to be uniform in $j$, and also in $\xi$. The same speech applies also for the quantity $R_{0}$. Finally, recall that all these quantities depend only on $K$ and $p$ appearing in hypothesis (6).

This having been noticed, let us define the approximate energy in Fourier variable, on each set $U \times I_{j}$ :

$$
E_{j, \varepsilon}(t, \xi):=S_{j, \varepsilon}(t, \xi) \widehat{u}(t, \xi) \cdot \widehat{u}(t, \xi) .
$$

Keep in mind that we have fixed $\varepsilon=1 /|\xi|$, but for convenience we will keep, for the moment, the notation with $\varepsilon$.

Recall also that we will work with $|\xi| \geq R_{0}>1$. However, in the case $|\xi| \leq R_{0}$ energy estimates immediately follow: it is enough to take the scalar product (in $\mathbb{R}^{m}$ ) of equation (15) with $\widehat{u}$ and to apply Gronwall's lemma.

We notice here that $E_{j, \varepsilon}(t, \xi) \sim|\widehat{u}(t, \xi)|^{2}$ for any $t$ and $|\xi| \geq R_{0}$, for some constants which are uniform in $(t, \xi)$.

Now, we differentiate the energy with respect to time. Using equation (14) and the properties stated in Lemma 1 and Proposition 1, we easily find

$$
\begin{aligned}
\partial_{t} E_{j, \varepsilon}= & 2 \operatorname{Re}\left(S_{j, \varepsilon} \widehat{L u} \cdot \widehat{u}\right)+ \\
& +2 \operatorname{Re}\left(-i S_{j, \varepsilon}\left(A-A_{\varepsilon}\right) \widehat{u} \cdot \widehat{u}\right)+R_{j, \varepsilon} \widehat{u} \cdot \widehat{u}+|\xi|^{-1} \partial_{t} S_{j, \varepsilon}^{1} \widehat{u} \cdot \widehat{u} .
\end{aligned}
$$

We collect here straightforward estimates of the previous terms. We have 


$$
\begin{aligned}
\left|2 \operatorname{Re}\left(S_{j, \varepsilon} \widehat{L u} \cdot \widehat{u}\right)\right| & \leq C|\widehat{\operatorname{Lu}}(t, \xi)|\left(E_{j, \varepsilon}(t, \xi)\right)^{1 / 2} \\
\left|2 \operatorname{Re}\left(-i S_{j, \varepsilon}\left(A-A_{\varepsilon}\right) \widehat{u} \cdot \widehat{u}\right)\right| & \leq C\left|A-A_{\varepsilon}\right|_{\mathscr{M}} E_{j, \varepsilon}(t, \xi) \\
\left|R_{j, \varepsilon} \widehat{u} \cdot \widehat{u}\right| & \leq C E_{j, \varepsilon}(t, \xi) \\
\left|\partial_{t} S_{j, \varepsilon}^{1} \widehat{u} \cdot \widehat{u}\right| & \leq C\left|\partial_{t} S_{j, \varepsilon}^{1}\right|_{\mathscr{M}} E_{j, \varepsilon} .
\end{aligned}
$$

Recall that both $A$ and $A_{\varepsilon}$ are homogeneous of degree 1 in $\xi$. Notice also that all the multiplicative constants are uniform in $t$ and $\xi$, and they just depend on $K$ and $p$.

Therefore, by (17) and the previous bounds, for each $j$ we infer the inequality

$$
\begin{aligned}
\partial_{t} E_{j, \varepsilon}(t, \xi) \leq C( & |\widehat{\operatorname{Lu}}(t, \xi)|\left(E_{j, \varepsilon}(t, \xi)\right)^{1 / 2}+ \\
& \left.+\left(1+\left|A-A_{\mathcal{\varepsilon}}\right|_{\mathscr{M}}+|\xi|^{-1}\left|\partial_{t} S_{j, \varepsilon}^{1}(t, \xi)\right|_{\mathscr{M}}\right) E_{j, \varepsilon}(t, \xi)\right) .
\end{aligned}
$$

Starting from this relation, if we define $e_{j, \varepsilon}(t, \xi):=\left(E_{j, \varepsilon}(t, \xi)\right)^{1 / 2}$ and

$$
\Phi_{j}(t, \xi):=C\left(1+|\xi|^{-1}\left|\partial_{t} S_{j, \varepsilon}^{1}(\tau, \xi)\right|_{\mathscr{M}}+\left|A(\tau, \xi)-A_{\mathcal{E}}(\tau, \xi)\right|_{\mathscr{M}}\right)
$$

Gronwall's inequality immediately entails, for any $t \in\left[t_{j}^{0}, t_{j}^{1}\right]$,

$$
e_{j, \varepsilon}(t, \xi) \leq e^{\int_{t_{j}^{t}}^{t} \Phi_{j}(\tau, \xi) d \tau} e_{j, \varepsilon}\left(t_{j}^{0}, \xi\right)+\int_{t_{j}^{0}}^{t} e^{\int_{\tau}^{t} \Phi_{j}(s, \xi) d s}|\widehat{L u}(\tau, \xi)| d \tau
$$

This inequality immediately implies, for all $t \in\left[t_{j}^{0}, t_{j}^{1}\right]$, the control

$$
|\widehat{u}(t, \xi)| \leq \frac{C_{2}}{C_{1}}\left(e^{\int_{t_{j}^{0}}^{t} \Phi_{j}(\tau, \xi) d \tau}\left|\widehat{u}\left(t_{j}^{0}, \xi\right)\right|+\int_{t_{j}^{0}}^{t} e^{\int_{\tau}^{t} \Phi_{j}(s, \xi) d s}|\widehat{L u}(\tau, \xi)| d \tau\right)
$$

(without loss of generality, we can suppose $C_{2} \geq 1$ ).

Now, let $\xi \neq 0$ fixed and $t \in[0, T]$. Let us set $j=\min \left\{k \mid t \in I_{k}\right\}$ (we know that the minimum is taken in a set of at most two elements, by construction). Then, we have estimate (19).

But $t_{j}^{0} \in I_{j-1}$ by our assumptions. So, we can replace the value of $\left|\widehat{u}\left(t_{j}^{0}, \xi\right)\right|$ in (19) by the corresponding estimate at level $j-1$ : we get

$$
\begin{aligned}
& |\widehat{u}(t, \xi)| \leq\left(\frac{C_{2}}{C_{1}}\right)^{2}\left(\exp \left(\int_{t_{j}^{0}}^{t} \Phi_{j} d \tau+\int_{t_{j-1}^{0}}^{t_{j}^{0}} \Phi_{j-1} d \tau\right)\left|\widehat{u}\left(t_{j-1}^{0}, \xi\right)\right|+\right. \\
& \left.\quad+\int_{t_{j-1}^{0}}^{t_{j}^{0}} \exp \left(\int_{t_{j}^{0}}^{t} \Phi_{j} d s+\int_{\tau}^{t_{j}^{0}} \Phi_{j-1} d s\right)|\widehat{L u}(\tau, \xi)| d \tau+\int_{t_{j}^{0}}^{t} e^{\int_{\tau}^{t} \Phi_{j} d s}|\widehat{L u}(\tau, \xi)| d \tau\right) .
\end{aligned}
$$

It is easy to combine the two terms in the last line to arrive at the inequality 


$$
\begin{aligned}
|\widehat{u}(t, \xi)| \leq\left(\frac{C_{2}}{C_{1}}\right)^{2} & \left(\exp \left(\int_{t_{j}^{0}}^{t} \Phi_{j} d \tau+\int_{t_{j-1}^{0}}^{t_{j}^{0}} \Phi_{j-1} d \tau\right)\left|\widehat{u}\left(t_{j-1}^{0}, \xi\right)\right|+\right. \\
& \left.+\int_{t_{j-1}^{0}}^{t} \exp \left(\int_{t_{j}^{0}}^{t} \Phi_{j} d s+\int_{\tau}^{t_{j}^{0}} \Phi_{j-1} d s\right)|\widehat{L u}(\tau, \xi)| d \tau\right) .
\end{aligned}
$$

At this point, a careful but straightforward induction allows us to conclude that

$$
\begin{aligned}
|\widehat{u}(t, \xi)| \leq & \left(\frac{C_{2}}{C_{1}}\right)^{J}\left(\exp \left(\int_{t_{j}^{0}}^{t} \Phi_{j} d \tau+\sum_{k=1}^{j-1} \int_{t_{k}^{0}}^{t_{k+1}^{0}} \Phi_{k} d \tau\right)|\widehat{u}(0, \xi)|+\right. \\
& \left.+\int_{t_{j-1}^{0}}^{t} \exp \left(\int_{t_{j}^{0}}^{t} \Phi_{j} d s+\sum_{k=1}^{j-1} \int_{t_{k}^{0}}^{t_{k+1}^{0}} \Phi_{k} d s+\int_{\tau}^{t_{1}^{0}} \Phi_{1} d s\right)|\widehat{L u}(\tau, \xi)| d \tau\right) .
\end{aligned}
$$

Now, our next goal is to bound the exponential terms in the previous estimate. First of all, we want to exploit the linearity of the time integral: this is a key point to get our precise energy estimates. Denoting by $\chi_{\mathscr{O}}$ the characteristic function of a set $\mathscr{O}$, we have

$$
\begin{aligned}
\int_{t_{j}^{0}}^{t} \Phi_{j} d \tau+\sum_{k=1}^{j-1} \int_{t_{k}^{0}}^{t_{k+1}^{0}} \Phi_{k} d \tau & =\int_{0}^{t}\left(\Phi_{j} \chi_{\left[t, t_{j}^{0}\right]}+\sum_{k=1}^{j-1} \Phi_{k} \chi_{\left[t_{k+1}^{0}, t_{k}^{0}\right]}\right) d \tau \\
\int_{t_{j}^{0}}^{t} \Phi_{j} d s+\sum_{k=1}^{j-1} \int_{t_{k}^{0}}^{t_{k+1}^{0}} \Phi_{k} d s+\int_{\tau}^{t_{1}^{0}} \Phi_{1} d s & =\int_{\tau}^{t}\left(\Phi_{j} \chi_{\left[t, t_{j}^{0}\right]}+\sum_{k=1}^{j-1} \Phi_{k} \chi_{\left[t_{k+1}^{0}, t_{k}^{0}\right]}\right) d s .
\end{aligned}
$$

In order to control the quantities which are inside the integrals, keep in mind definition (18) of the functions $\Phi_{k}$. Of course, the constant 1 does not give any complication; so, let us focus on the other terms.

Let us set $\theta=1 / p^{\prime}=1-1 / p$. We know that, for any $k$ and any $t^{\prime} \in\left[t_{k}^{0}, t_{k}^{1}\right]$, Hölder's inequality allows us to get

$$
\begin{aligned}
|\xi|^{-1} \int_{t_{k}^{0}}^{t^{\prime}}\left|\partial_{t} S_{k, \varepsilon}^{1}(\tau, \xi)\right|_{\mathscr{M}} d \tau \leq C|\xi|^{-1}\left(t^{\prime}-t_{k}^{0}\right)^{\theta}\left\|\partial_{t} S_{k, \mathcal{\varepsilon}}^{1}(\cdot, \xi)\right\|_{L^{p}\left(\left[t_{k}^{0}, t_{k}^{1}\right] ; \mathscr{M}\right)} \\
\int_{t_{k}^{0}}^{t^{\prime}}\left|A-A_{\mathcal{\varepsilon}}\right|_{\mathscr{M}} d \tau \leq C|\xi|\left(t^{\prime}-t_{k}^{0}\right)^{\theta} \sup _{1 \leq j \leq n}\left\|A_{j}-A_{j, \varepsilon}\right\|_{L^{p}\left(\left[t_{k}^{0}, t_{k}^{1}\right] ; \mathscr{M}\right)} .
\end{aligned}
$$

Recall that the constants appearing in the inequalities here above depend just on $K$ and $p$ of condition (6). Then, they are uniform with respect to $k \in[1, J]$ and also with respect to $\xi$, since $J=J(\xi) \leq J_{M}$. On the other hand, the $L^{p}$ norms can be bounded as done in [11] (see Proposition 3.22 of that paper): 
A few remarks on hyperbolic systems with Zygmund in time coefficients

$$
\begin{aligned}
\left\|\partial_{t} S_{k, \varepsilon}^{1}(\cdot, \xi)\right\|_{L^{p}\left(\left[t_{k}^{0}, t_{k}^{1}\right] ; \mathscr{M}\right)} & \leq C|\xi| \log ^{\ell}(1+|\xi|) \\
\sup _{1 \leq j \leq n}\left\|A_{j}-A_{j, \varepsilon}\right\|_{L^{p}\left(\left[t_{k}^{0}, t_{k}^{1}\right] ; \mathscr{M}\right)} & \leq \sup _{1 \leq j \leq n}\left\|A_{j}-A_{j, \varepsilon}\right\|_{L^{p}([0, T] ; \mathscr{M})} \\
& \leq \frac{C}{|\xi|} \log ^{\ell}(1+|\xi|),
\end{aligned}
$$

where, as usual, $\ell=0$ under the $\mathscr{Z}_{p}$ hypothesis and $\ell=1$ in the $\mathscr{L} \mathscr{Z}_{p}$ case. For simplicity of exposition, from now on we fix the choice $\ell=1$.

Therefore, let us come back to the exponential terms in (20). Thanks to the linearity of the integral, we deduce the following inequalities:

$$
\int_{0}^{t}\left(\Phi_{j} \chi_{\left[t, t_{j}^{0}\right]}+\sum_{k=1}^{j-1} \Phi_{k} \chi_{\left[t_{k+1}^{0}, t_{k}^{0}\right]}\right) d \tau \leq t+C t^{\theta} \log (1+|\xi|)
$$

for the former term, while for the latter

$$
\int_{\tau}^{t}\left(\Phi_{j} \chi_{\left[t, t_{j}^{0}\right]}+\sum_{k=1}^{j-1} \Phi_{k} \chi_{\left[t_{k+1}^{0}, t_{k}^{0}\right]}\right) d s \leq(t-\tau)+C(t-\tau)^{\theta} \log (1+|\xi|) .
$$

Putting these controls into (20), we finally get, for all $t \in[0, T]$ and all $|\xi| \geq R_{0}$,

$$
|\widehat{u}(t, \xi)| \leq C e^{C t}\left(|\xi|^{\widetilde{\beta} t^{\theta}}|\widehat{u}(0, \xi)|+\int_{0}^{t}|\xi|^{\widetilde{\beta}(t-\tau)^{\theta}}|\widehat{L u}(\tau, \xi)| d \tau\right)
$$

for some suitable positive constants $C$ and $\widetilde{\beta}$, depending only on $p$ and $K$ appearing in (6). At this point, we have just to multiply both sides by $|\xi|^{-\widetilde{\beta} t^{\theta}}$ and to take the $L^{2}$ norm (or the $H^{s}$ norm, for any $s$ ) with respect to $\xi$.

The proof of the energy estimates is then completed.

\section{Proof of the finite propagation speed property}

The present section is devoted to the proof of Theorem 2. For the sake of clarity of exposition, we avoid the technicalities needed to treat the problem globally in time (recall Subsection 3.4). Hence, we suppose that $[0, T]$ is covered by just one interval $I_{1}$, and that we have a unique symmetrizer there.

Still, the result of [20] does not apply in a direct way. As a matter of fact, we do not have available a microlocal symmetrizer for our system: we can just construct approximate symmetrizers for the perturbed operator $L_{\mathcal{\varepsilon}}$ (see Proposition 1). Moreover, we have to check that no troubles are produced in the estimates by the terms which originate from the remainders $S_{\varepsilon}^{1}$.

The main tool we use to establish finite propagation speed is Paley-Wiener Theorem, which we recall here. The present form is the one stated in [8], Section 1. 
Theorem 5. (I) Let $u \in \mathscr{H}^{\prime}$ and $r>0$. Then $u \in \mathscr{A}^{\prime}$, with supp $u \subset B(r)$, if and only if the following condition is verified: for all $\delta>0$, there exists a constant $C_{\delta}>0$ such that

$$
|\widehat{u}(\zeta)| \leq C_{\delta} \exp (\delta|\xi|+(r+\delta)|\eta|)
$$

for all $\zeta=\xi+i \eta \in \mathbb{C}^{n}$, with $|\zeta| \geq 1$.

(II) Let $u \in L^{1}\left([0, T] ; \mathscr{A}^{\prime}\right)$, with support contained in the ball $B(r)$. Then, for all $\delta>0$, there exists a constant $C_{\delta}>0$ such that

$$
\int_{0}^{T}|\widehat{u}(t, \zeta)| d t \leq C_{\delta} \exp (\delta|\xi|+(r+\delta)|\eta|)
$$

for all $\zeta=\xi+i \eta \in \mathbb{C}^{n}$, with $|\zeta| \geq 1$.

In the statement above, we have adopted the notations which have been introduced in Subsection 3.1.

\subsection{A key estimate}

Thanks to Theorem 5, the first step of the proof to Theorem 2 consists in establishing a fundamental bound on the growth of the solution in Fourier variables.

More precisely, we are going to show the next result, still for the case $\ell=1$ (the analogue for $\ell=0$ presents no special differences).

Lemma 2. Let us consider the first-order system (4), and let us assume it to be hyperbolic with constant multiplicities. Suppose moreover that the coefficients $\left(A_{j}\right)_{1 \leq j \leq n}$ satisfy condition (6), for some $1<p \leq+\infty$ and for $\ell=1$. Fixed $s \in \mathbb{R}$, assume that $u_{0} \in H^{s}$ and $f \in L^{1}\left([0, T] ; H^{s}\right)$, and let $u$ be the solution to problem (8) belonging to $\mathscr{C}\left([0, T] ; H^{s-\beta(\cdot)}\right)$.

Finally, for $\zeta=\xi+i \eta \in \mathbb{C}^{n}$, define the function

$$
\varphi_{\varepsilon}(t, \zeta):=\mathbb{K}\left(1+|\xi|^{-1}\left|\partial_{t} S_{\varepsilon}^{1}(t, \xi)\right|_{\mathscr{M}}+\left|A(t, \xi)-A_{\varepsilon}(t, \xi)\right|_{\mathscr{M}}+\alpha(t)|\eta|\right),
$$

where $\alpha$ is the quantity defined in the statement of Theorem $2,\left(S_{\varepsilon}\right)_{\varepsilon}$ is the family of smooth approximate symmetrizers (constructed in Paragraph 3.3.1), and $\mathbb{K}>0$ is a large enough constant, depending only on $K$ appearing in (6).

Then there exists a constant $C>0$ such that the estimate

$$
|\widehat{u}(t, \zeta)| \leq C \exp \left(\int_{0}^{t} \varphi_{\varepsilon}(\tau, \zeta) d \tau\right)\left(\left|\widehat{u}_{0}(\zeta)\right|+\int_{0}^{T}|\widehat{f}(\tau, \zeta)| d \tau\right) .
$$

holds true for all $t \in[0, T]$ and all $\zeta=\xi+i \eta$ satisfying $|\zeta| \geq 1$.

We split the proof of the previous statement in high and low frequencies estimates. The meaning of the words "high" and "low" here will be clarified in a while: it depends essentially on property $(b)$ established in Proposition 1. 
Remark 9. The constant $C$ appearing in the estimate above depends on $K$ and the index $p$ which both appear in (6), and on the time $T$.

\subsubsection{High frequencies estimates}

As said in the previous statement, let $\left(S_{\varepsilon}(t, \xi)\right)_{\varepsilon}$ be the family of smooth approximate symmetrizers to our system, and let us define an approximate energy for $u$ in Fourier variables: for $\zeta=\xi+i \eta$, we set

$$
E_{\varepsilon}(t, \zeta):=S_{\varepsilon}(t, \xi) \widehat{u}(t, \zeta) \cdot \widehat{u}(t, \zeta) .
$$

We remark that it is important to keep $S_{\varepsilon}$ depending just on $\operatorname{Re} \zeta=\xi$, not to lose self-adjointness ${ }^{1}$ and symmetrizability ${ }^{2}$ properties.

Let us fix $\varepsilon=|\xi|^{-1}$, as in Paragraph 3.3.1, and let $R_{0}$ be the positive real number given by Proposition 1 . From now on, let us work for $|\xi| \geq R_{0}$ : low frequencies estimates will be shown later on (see Paragraph 4.1.2 below).

Let us recall that, for all $(t, \zeta) \in \mathbb{R} \times \mathbb{C}^{n}$, with $\zeta=\xi+i \eta$ and $|\xi| \geq R_{0}$, one has

$$
C_{1}|\widehat{u}(t, \zeta)|^{2} \leq E_{\varepsilon}(t, \zeta) \leq C_{2}|\widehat{u}(t, \zeta)|^{2} .
$$

Next, let us compute the time derivative of the approximated energy $E_{\varepsilon}$. Keep in mind that, from equation $L u=f$, if we pass in the Phase Space we get

$$
\partial_{t} \widehat{u}(t, \zeta)+i A(t, \zeta) \widehat{u}(t, \zeta)=\widehat{f}(t, \zeta),
$$

where, by (5), we have $A(t, \zeta)=A(t, \xi)+i A(t, \eta)$. Therefore, differentiating (24) with respect to time we find

$$
\begin{aligned}
\partial_{t} E_{\varepsilon} & =\partial_{t} S_{\varepsilon} \widehat{u} \cdot \widehat{u}+2 \operatorname{Re}\left(S_{\varepsilon} \partial_{t} \widehat{u} \cdot \widehat{u}\right) \\
& =\partial_{t} S_{\varepsilon} \widehat{u} \cdot \widehat{u}+2 \operatorname{Re}\left(S_{\varepsilon} \widehat{f} \cdot \widehat{u}\right)+2 \operatorname{Re}\left(-i S_{\varepsilon} A \widehat{u} \cdot \widehat{u}\right) .
\end{aligned}
$$

Recall that, in the last term, $S_{\varepsilon}=S_{\varepsilon}(t, \xi)$ while $A=A(t, \zeta)$. Keeping in mind formula (13), straightforward computations lead us to

$$
\begin{aligned}
\partial_{t} E_{\varepsilon}= & \partial_{t} S_{\varepsilon}^{0}(t, \xi) \widehat{u} \cdot \widehat{u}+|\xi|^{-1} \partial_{t} S_{\varepsilon}^{1}(t, \xi) \widehat{u} \cdot \widehat{u}+ \\
& +2 \operatorname{Re}\left(S_{\varepsilon} \widehat{f} \cdot \widehat{u}\right)+2 \operatorname{Re}\left(-i S_{\varepsilon}(t, \xi) A_{\varepsilon}(t, \xi) \widehat{u} \cdot \widehat{u}\right)+ \\
& +2 \operatorname{Re}\left(-i S_{\varepsilon}(t, \xi)\left(A(t, \xi)-A_{\varepsilon}(t, \xi)\right) \widehat{u} \cdot \widehat{u}\right)+2 \operatorname{Re}\left(S_{\varepsilon}(t, \xi) A(t, \eta) \widehat{u} \cdot \widehat{u}\right) .
\end{aligned}
$$

\footnotetext{
${ }^{1}$ For instance, already for $m=1$, take the function $S(\xi)=\xi /|\xi|$ : then $S(\zeta)$ is well-defined, but it is no more self-adjoint.

${ }^{2}$ We know that $S(t, \xi)$ is a symmetrizer for $A(t, \xi)$, but this does not imply that $S(t, \zeta)$ is a symmetrizer for $A(t, \zeta)=A(t, \xi)+i A(t, \eta)$.
} 
We start estimating each term in the right-hand side of the last equation. In the inequalities we are going to present here below, the multiplicative constants, which we generically denote by $C$, just depend on $K$ appearing in condition (6).

First of all, it is easy to get, for a constant $C>0$ (depending on the $L^{\infty}$ norm of the symmetrizer, so in the end on $K$ ), the bound

$$
\left|2 \operatorname{Re}\left(S_{\varepsilon} \widehat{f} \cdot \widehat{u}\right)\right| \leq C|\widehat{f}|\left(E_{\varepsilon}\right)^{1 / 2} .
$$

Moreover, we immediately have the following inequalities:

$$
\begin{aligned}
\left.|| \xi\right|^{-1} \partial_{t} S_{\varepsilon}^{1}(t, \xi) \widehat{u} \cdot \widehat{u} \mid & \leq C|\xi|^{-1}\left|\partial_{t} S_{\varepsilon}^{1}(t, \xi)\right|_{\mathscr{M}} E_{\varepsilon} \\
\left|\operatorname{Re}\left(S_{\varepsilon}(t, \xi) A(t, \eta) \widehat{u} \cdot \widehat{u}\right)\right| & \leq C \sum_{j}\left|A_{j}(t)\right|_{\mathscr{M}}|\eta| E_{\varepsilon} \\
\left|\operatorname{Re}\left(-i S_{\varepsilon}(t, \xi)\left(A(t, \xi)-A_{\mathcal{\varepsilon}}(t, \xi)\right) \widehat{u} \cdot \widehat{u}\right)\right| & \leq C\left|A(t, \xi)-A_{\varepsilon}(t, \xi)\right|_{\mathscr{M}} E_{\varepsilon} .
\end{aligned}
$$

As for the last two terms,

$$
\partial_{t} S_{\varepsilon}^{0}(t, \xi) \widehat{u} \cdot \widehat{u}+2 \operatorname{Re}\left(-i S_{\varepsilon}(t, \xi) A_{\varepsilon}(t, \xi) \widehat{u} \cdot \widehat{u}\right),
$$

we exploit again formula (13). Since $S_{\mathcal{\varepsilon}}^{0}(t, \xi) A_{\mathcal{\varepsilon}}(t, \xi)$ is self-adjoint and Lemma 1 holds true, we deduce that the previous sum is equal to $R_{\varepsilon} \widehat{u} \cdot \widehat{u}$, with $\left(R_{\varepsilon}\right)_{\varepsilon}$ uniformly bounded in $L^{\infty}([0, T])$. Hence we find

$$
\left|\partial_{t} S_{\varepsilon}^{0}(t, \xi) \widehat{u} \cdot \widehat{u}+2 \operatorname{Re}\left(-i S_{\varepsilon}(t, \xi) A_{\varepsilon}(t, \xi) \widehat{u} \cdot \widehat{u}\right)\right| \leq C E_{\varepsilon} .
$$

Putting inequalities from (27) to (31) all together, we infer

$$
\partial_{t} E_{\varepsilon}(t, \zeta) \leq C\left(|\widehat{f}(t, \zeta)|\left(E_{\varepsilon}(t, \zeta)\right)^{1 / 2}+\varphi_{\varepsilon}(t, \zeta) E_{\varepsilon}(t, \zeta)\right) .
$$

Now, if we define $e_{\varepsilon}(t, \zeta)=\left(E_{\varepsilon}(t, \zeta)\right)^{1 / 2}$, an application of Gronwall Lemma to the previous inequality implies

$$
e_{\varepsilon}(t, \zeta) \leq e^{\int_{0}^{t} \varphi_{\varepsilon}(\tau, \zeta) d \tau}\left(e_{\varepsilon}(0, \zeta)+\int_{0}^{t}|\widehat{f}(\tau, \zeta)| d \tau\right)
$$

which immediately allows us to obtain estimate (23), after taking (25) into account.

Then, Lemma 2 is proved in the case of $|\operatorname{Re} \zeta| \geq R_{0}$.

\subsubsection{Low frequencies estimate}

Let us now deal with the "low frequency" case, i.e. when $|\zeta| \geq 1$ but $|\operatorname{Re} \zeta| \leq R_{0}$.

In this instance, getting inequality (23) is actually easier. Indeed, multiplying equation (26) by $\widehat{u}(t, \zeta)$ and applying Gronwall lemma again, we deduce 


$$
\begin{aligned}
|\widehat{u}(t, \zeta)| & \leq\left(\left|\widehat{u}_{0}(\zeta)\right|+\int_{0}^{t}|\widehat{f}(\tau, \zeta)| d \tau\right) \exp \left(\int_{0}^{t}(|A(\tau, \xi)| \mathscr{M}+|A(\tau, \eta)| \mathscr{M}) d \tau\right) \\
& \leq e^{c(T) R_{0}}\left(\left|\widehat{u}_{0}(\zeta)\right|+\int_{0}^{t}|\widehat{f}(\tau, \zeta)| d \tau\right) \exp \left(\int_{0}^{t}|A(\tau, \eta)|_{\mathscr{M}} d \tau\right),
\end{aligned}
$$

where the constant $c(T)$ appears thanks to (7); it depends on $T$, and also on $p$ and $K$. Therefore, (23) immediately follows.

\subsection{End of the proof of Theorem 2}

Let us come back to the proof of the finite propagation speed.

Our starting point is the inequality established in Lemma 2 above. By our hypotheses, combining it with Theorem 5 , we obtain the following property: for any $\delta>0$ fixed, there exists a constant $C_{\delta}>0$ such that

$$
|\widehat{u}(t, \zeta)| \leq C C_{\delta} \exp \left(\int_{0}^{t} \varphi_{\varepsilon}(\tau, \zeta) d \tau\right) \exp \left(\delta|\xi|+\left(r_{0}+\delta\right)|\eta|\right)
$$

for any $\zeta=\xi+i \eta \in \mathbb{C}^{n}$, with $|\zeta| \geq 1$. So, our next goal is to find suitable estimates for the integral inside the exponential term. Recall the definition of $\varphi_{\varepsilon}$, namely

$$
\varphi_{\varepsilon}(t, \zeta):=\mathbb{K}\left(1+|\xi|^{-1}\left|\partial_{t} S_{\mathcal{\varepsilon}}^{1}(t, \xi)\right|_{\mathscr{M}}+\left|A(t, \xi)-A_{\varepsilon}(t, \xi)\right|_{\mathscr{M}}+\alpha(t)|\eta|\right) .
$$

The strategy is the same as in Subsection 3.4: namely, we exploit Hölder inequality to make the $L^{p}$ norm in time appear, and the we use Proposition 3.22 of [11]. More precisely, arguing as done in (21) and in (22) respectively, we deduce

$$
\begin{aligned}
\int_{0}^{t}|\xi|^{-1}\left|\partial_{t} S_{\mathcal{\varepsilon}}^{1}(\tau, \xi)\right|_{\mathscr{M}} d \tau & \leq C t^{\theta} \log (1+|\xi|) \\
\int_{0}^{t}\left|A(\tau, \xi)-A_{\mathcal{E}}(\tau, \xi)\right|_{\mathscr{M}} d \tau & \leq C t^{\theta} \log (1+|\xi|),
\end{aligned}
$$

where $\theta=1-1 / p$ as before, and the constants $C$ depend just on $K$ and $p$.

Thanks to the previous controls, we find, for new suitable constants $C$ and $C_{\delta}$, possibly depending also on $T$,

$$
\begin{aligned}
|\widehat{u}(t, \zeta)| & \leq C_{\delta} \exp (C \log (1+|\xi|)) \exp (\delta|\xi|+(r(t)+\delta)|\eta|) \\
& \leq C_{\delta} \mathscr{E}_{\delta}(\xi) \exp (2 \delta|\xi|+(r(t)+2 \delta)|\eta|)
\end{aligned}
$$

where we have defined

$$
\mathscr{E}_{\delta}(\xi):=\exp (C \log (1+|\xi|)-\delta|\xi|) .
$$


Notice that the function $\mathscr{E}_{\delta}(\xi)$ is bounded for $\xi \in \mathbb{R}^{n}$, for all $\delta>0$ fixed. Hence the previous inequality becomes

$$
|\widehat{u}(t, \zeta)| \leq \widetilde{C}_{\delta} \exp (2 \delta|\xi|+(r(t)+2 \delta)|\eta|) .
$$

Keeping in mind Theorem 5, this estimate concludes the proof of Theorem 2.

Acknowledgements The author whishes to express all his gratitude to T. Alazard, whose relevant question motivated the study presented in this note about finite propagation speed.

$\mathrm{He}$ is also deeply grateful to the anonymous referee for the careful reading and the constructive remarks, which helped to improve the presentation of the paper.

\section{References}

1. Ambrosio, L.: Transport equation and Cauchy problem for $B V$ vector fields. Invent. Math., 158, 227-260 (2004)

2. Avellaneda, M., Bardos, C., Rauch, J.: Contrôlabilité exacte, homogénéisation et localisation d'ondes dans un milieu non-homogène. Asymptotic Anal., 5, 481-494 (1992)

3. Bahouri, H., Chemin, J.-Y.: Équations de transport relatives à des champs de vecteurs nonlipschitziens et mécanique des fluides. Arch. Ration. Mech. Anal., 127, 159-181 (1994)

4. Bahouri, H., Chemin, J.-Y., Danchin, R.: Fourier Analysis and Nonlinear Partial Differential Equations. Grundlehren der Mathematischen Wissenschaften (Fundamental Principles of Mathematical Sciences), Springer, Heidelberg (2011)

5. Castro, C., Zuazua, E.: Concentration and lack of observability of waves in highly heterogeneous media. Arch. Ration. Mech. Anal., 164, 39-72 (2002)

6. Chemin, J.-Y.: Fluides parfaits incompressibles. Astérisque, 230 (1995)

7. Cicognani, M., Colombini F.: Modulus of continuity of the coefficients and loss of derivatives in the strictly hyperbolic Cauchy problem. J. Differential Equations, 221, 143-157, (2006)

8. Colombini, F., De Giorgi, E., Spagnolo, S.: Sur les équations hyperboliques avec des coefficients qui ne dépendent que du temps. Ann. Scuola Normale Sup. Pisa Cl. Scienze (4), 6, 511-559 (1979)

9. Colombini, F., Del Santo, D., Fanelli, F., Métivier, G.: Time-dependent loss of derivatives for hyperbolic operators with non-regular coefficients. Comm. Partial Differential Equations, 38, 1791-1817, (2013)

10. Colombini, F., Del Santo, D., Fanelli, F., Métivier, G.: A well-posedness result for hyperbolic operators with Zygmund coefficients. J. Math. Pures Appl. (9), 100, 455-475 (2013)

11. Colombini, F., Del Santo, D., Fanelli, F., Métivier, G.: The well-posedness issue in Sobolev spaces for hyperbolic systems with Zygmund-type coefficients. Comm. Partial Differential Equations, 40, 2082-2121 (2015)

12. Colombini, F., Del Santo, D., Fanelli, F., Métivier, G.: On the Cauchy problem for microlocally symmetrizable hyperbolic systems with log-Lipschitz coefficients. In preparation (2016)

13. Colombini, F., Lerner, N.: Hyperbolic operators with non-Lipschitz coefficients. Duke Math. J., 77, 657-698 (1995)

14. Colombini, F., Métivier, G.: The Cauchy problem for wave equations with non-Lipschitz coefficients; application to continuation of solutions of some nonlinear wave equations. Ann. Sci. École Norm. Sup. (4), 41, 177-220 (2008)

15. Colombini, F., Métivier, G.: Couterexamples to the well-posedness of the Cauchy problem for hyperbolic systems. Anal. PDE, 8, 499-511 (2015) 
16. Danchin, R.: On the well-posedness of the incompressible density-dependent Euler equations in the $L^{p}$ framework. J. Differential Equations, 248, 2130-2170 (2010)

17. Danchin, R., Fanelli, F.: The well-posedness issue for the density-dependent Euler equations in endpoint Besov spaces, J. Math. Pures Appl., 96, 253-278 (2011)

18. Delort, J.-M.: Existence de nappes de tourbillon en dimension deux. J. Amer. Math. Soc., 4, 553-586 (1991)

19. Di Perna, R.-J., Lions, P.-L.: Ordinary differential equations, transport theory and Sobolev spaces. Invent. Math., 98, 511-547 (1989)

20. Fanelli, F.: Some local questions for hyperbolic systems with non-regular time dependent coefficients. Submitted (2015)

21. Fanelli, F., Zuazua, E.: Weak observability estimates for 1-D wave equations with rough coefficients. Ann. Inst. H. Poincaré Anal. Non Linéaire, 32, 245-277 (2015)

22. Feireisl, E., Gallagher, I., Gérard-Varet, D., Novotný, A.: Multi-scale analysis of compressible viscous and rotating fluids. Comm. Math. Phys., 314, 641-670 (2012)

23. Fernández-Cara, E., Zuazua, E.: On the null controllability ot the one-dimensional heat equation with BV coefficients. Comput. Appl. Math., 21, 167-190 (2002)

24. Gallagher, I., Saint-Raymond, L.: Weak convergence results for inhomogeneous rotating fluid equations. J. Anal. Math., 99, 1-34 (2006)

25. Ivriı̌, V. J., Petkov, V. M.: Necessary conditions for the Cauchy problem for non-strictly hyperbolic equations to be well-posed. Usephi Math. Nauk, 29, 3-70 (1974). English transl. Russian Math. Surveys, 29, 1-70 (1974)

26. Joly, J.-L., Métivier, G., Rauch, J.: Hyperbolic domains of determinacy and Hamilton-Jacobi equations. J. Hyperbolic Differ. Equ., 2, 713-744 (2005)

27. Métivier, G.: Para-differential calculus and applications to the Cauchy problem for nonlinear systems. Centro di Ricerca Matematica "Ennio De Giorgi” (CRM) Series, Edizioni della Normale, Pisa (2008)

28. Métivier, G.: $L^{2}$ well-posed Cauchy problems and symmetrizability of first order systems. J. Éc. Polytech. Math., 1, 39-70 (2014)

29. Métivier, G., Schochet, S.: The incompressible limit of the non-isentropic Euler equations. Arch. Ration. Mech. Anal., 158, 61-90 (2001)

30. Rauch, J.: Precise finite speed with bare hands. Methods Appl. Anal., 12, 267-277 (2005)

31. Tarama, S.: Energy estimate for wave equations with coefficients in some Besov type class. Electron. J. Differential Equations, Paper No. 85 (2007)

32. Treves, F.: Basic linear partial differential equations. Pure and Applied Mathematics, Academic Press, New York-London (1975)

33. Waters, A.: Multi-dimensional low regularity observability estimates. Submitted, arXiv preprint http: / /arxiv.org/abs/1512.02180 (2015)

34. Yudovich, V. I.: Non-stationary flows of an ideal incompressible fluid. Z̆. Vyčisl. Mat. i Mat. Fiz., 3, 1032-1066 (1963) 